\title{
Picture perception: An analysis of visual compensation
}

\author{
RICHARD R. ROSINSKI \\ University of Pittsburgh, Pittsburgh, Pennsylvania 15260 \\ TIMOTHY MULHOLLAND \\ Universidade de Brasilia, 70910 Brasilia, D.F., Brazil \\ DOUGLAS DEGELMAN \\ Eastern Nazarene College, Wollaston, Massachusetts 02170 \\ and \\ JAMES FARBER \\ New School for Social Research, New York, New York 10011
}

\begin{abstract}
Although geometric information is altered when a picture's viewing point is changed, such changes often do not affect perception. Two experiments assessed pictorial perception relative to the distortions introduced by viewing point dislocation. Results provide a psychophysical demonstration of pictorial compensation and suggest that it is based on the discrepancy between the actual and an assumed-correct viewing position. An explanation of pictorial compensation is offered that could be applied to direct picture perception and to picture-in-apicture perception.
\end{abstract}

A fundamental problem in the study of picture perception involves explaining how two-dimensional pictures and photographs are capable of producing accurate impressions of three-dimensional spatial layouts. This representational nature of pictures has been explained by some theorists (e.g., Gibson, 1954, 1971; Hochberg, 1962; Kennedy, 1974) in terms of the information available in the optical projection of a photograph. Under "ideal" conditions, a picture can project to the eye a near simulation of the scene it represents. Since there are similarities between the optic arrays projected from a picture and from the real scene, pictorial perception could involve the processing of the same visual information as does the perception of real scenes or objects.

However, this ideal case is rarely achieved or approximated. If a picture is viewed from the correct station point (corresponding to the geometric center of projection), it projects an array that is geometrically identical to one from the original scene. There are, however, an infinite number of other viewing points at which geometric relations are transformed. Consequently, there may be striking geometrical distortions in the array that is projected to the viewer's eye.

As Haber (1978) points out, the perceptual effects of such distortions are of considerable theoretical importance. If the perception of space in pictures depends solely on the geometric information projected to the viewer, viewing a picture from an incorrect point should affect the perception of depicted layout. The absence of perceptual effects resulting from displacing the viewing point may indicate that similarity between pictorial and environmental arrays is not the basic determiner of pictorial perception.

It has often been pointed out (cf. Pirenne, 1970) on the basis of causal or phenomenological observation that distortions of the pictorial array do not affect perception. As we walk past a painting or photograph, we perceive, apparently accurately, what is depicted in the picture. Pictured space does not appear to distort as the viewer changes position. The existence of such a phenomenon has suggested to some (e.g., Pirenne, 1970) that the perception of pictures must involve a compensation process that enables picture viewers to discount the effects of projective transformations on depicted space. However, little evidence exists for such a process-in part, because of the nature of prior research.

For example, let us point out that phenomenological data are of questionable validity here. The fact that people are not consciously aware of distortions does not imply that distortions go unregistered. There is a logical difficulty involved in being aware of distortion. Under ideal conditions, a pictorial array and an environmental array are identical. A distortion means that the pictorial array projected to the new viewing point has been transformed; that is, it no 
longer corresponds to the environmental array. Judgments of distortion are, in principle, impossible unless the picture viewer has independent specification of the environmental array.

Perkins (1973) has provided a rigorous, experimental demonstration of pictorial compensation for distortions of rectangularity. In general, however, few studies of compensation exist. One difficulty has been that in order to determine whether pictorial compensation exists, it is necessary to compare perceived space with both projected and actual spatial layout. Failure to make such comparisons may lead to inadequate stimulus control (cf. Hagen, 1974; Rosinski, 1976). Until recently, an analysis of pictorial transformations has not been generally available. Farber and Rosinski (1978) and Rosinski and Farber (1980) have provided a general geometric analysis of the effects of all dislocations of viewing point on projected space. In the present experiments, we have used this analysis to determine appropriate transforms of pictorial space. Our tasks required participants to make judgments of the slant of a surface depicted in photographs when the photographs were viewed from various points. In all conditions, viewing was from the same distance to the center of the picture but along lines of sight that intersected the picture plane at various angles. The optically projected slant of a surface depicted in a slanted photograph is given by the relation:

$$
\sin \theta^{\prime}=\sin \theta \frac{\sin \delta}{\sqrt{1+\cos \delta \sin 2 \theta}}
$$

where $\theta^{\prime}$ is the projected surface slant, $\theta$ is the depicted surface slant, and $\delta$ is the inclination of the picture plane.

The compensation question therefore reduces to two comparisons: To what extent does perceived slant correspond to projected slant, $\theta^{\prime}$, or to what extent does perceived slant correspond to depicted slant, $\theta$, regardless of picture plane orientation, $\delta$.

\section{EXPERIMENT 1}

In order to determine the effects of geometric distortion, we assessed judged orientation of a pictured surface when the picture was held frontally or was rotated. To evaluate the effects of distortion of perspective independently of other factors, the first experiment attempted to minimize all information except that provided by linear perspective.

\section{Method}

Observers. Ten students (five male, five female) from the University of Pittsburgh took part in the experiment in order to satisfy a course requirement. All individuals had visual acuity of $20 / 40$ (corrected) or better, and any who normally wore corrective lenses did so in the experiment.
Apparatus. Stimulus displays were constructed by photographing a square wave, black and white striped surface with a $4 \times 5$ view camera and $215-\mathrm{mm}$ lens. The optical slant around a vertical axis was varied over the range from 30 to $150 \mathrm{deg}$ (with $90 \mathrm{deg}=$ frontal, and slants greater than $90 \mathrm{deg}$ indicating the left side closer) in 10-deg increments by manipulating the angular relationship between the plane of the surface and the film plane. When the photographs were taken, the distance between the lens node and film plane axis was $250 \mathrm{~mm}$. Prints were $2 \times$ magnification of the negatives in which each stripe subtended approximately $.57 \mathrm{deg}$ at the axis of rotation. The photographs were cut into trapezoidal shapes so that outline perspective of the photograph and linear perspective of the grating bars were equivalent. They were mounted on flat black matte board.

In the study, the photographs were inserted into a viewing box. The interior of the viewing box was painted flat black and contained a $40-\mathrm{W}$ bulb for illumination. Cross-polarized filters eliminated reflections from the surface of the photograph and resulted in a luminance of $.17 \mathrm{~cd} / \mathrm{m}^{2}$ for the black stripes and $3.24 \mathrm{~cd} / \mathrm{m}^{2}$ for the white stripes.

The photographs were viewed monocularly through a $1-\mathrm{cm}$ aperture from one of two viewing points located $50 \mathrm{~cm}$ away from the center of the photo. The photographs projected linear perspective gradient information for surface slant around a vertical axis. From the correct viewing point, a line from the viewing point to the axis was normal to the photographic surface $(\delta=90 \mathrm{deg})$, and from the incorrect point, it was inclined ( $\delta=135 \mathrm{deg})$.

Each subject responded by setting the inclination of a vertically pivoted palm board with his or her right hand to match and slant of the surface depicted in the photograph. No attempt was made to conceal from the observers that they were viewing photographs, although they were required to move away from the aperture when photographs were being changed between trials. Judgments were recorded to the nearest degree. The subjects made judgments in both conditions in counterbalanced order. In each condition, they made judgments of 13 slants presented in two randomly ordered blocks.

\section{Results and Discussion}

The optical projection of each of the depicted surface slants for the two viewing conditions (derived from Equation 1) is presented in Figure 1. From the correct viewing point $(\delta=90 \mathrm{deg})$, projected slant always is equal to the depicted surface slant. From the incorrect point $(\delta=135 \mathrm{deg})$, projected slant is always greater than the depicted slant of the surface.

The important questions are whether judgments are determined by the geometry of the array and thus correspond to projected slant (the no-compensation hypothesis) or whether the visual system discounts the effects of distortion (the compensation hypothesis). Two kinds of evidence bear on these questions. First, as Equation 1 shows, any depicted surface orientation projects arrays corresponding to different optical slants when viewed from various locations. Therefore, condition differences in judged slant as a function of depicted surface slant indicate no compensation. Conversely, the absence of condition differences would indicate that the distorting effects of dislocating the viewing point are overcome by the visual system. Second, the absence of condition differences as a function of projected slant would indicate that judgments are controlled by the projection. The presence of such effects would show that distortions 


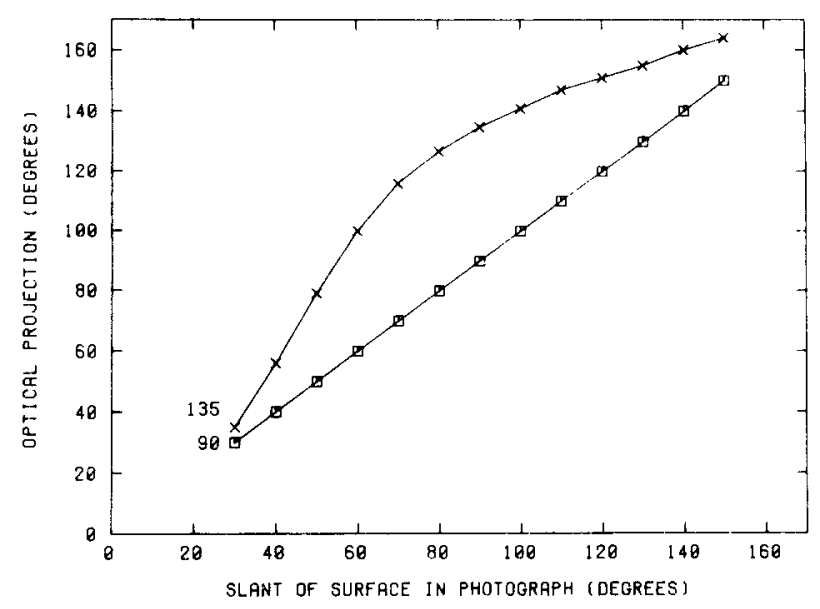

Figure 1. Projected orientation of the surface in the photograph when the photo is viewed from the correct $(\delta=90 \mathrm{deg})$ and the incorrect $(\delta=135 \mathrm{deg})$ viewing points.

of the projection do not affect perception. Thus, the existence of compensation can be determined by evaluating the extent to which geometric distortions are reflected in performance.

The data, depicted in Figure 2, indicate that little if any compensation occurred. Within-cell variances were homogeneous, and judgments were analyzed with a 2 (sex) by 2 (condition) by 2 (block) by 13 (slant) ANOVA with repeated measures on the last three factors. The effect of condition was significant $[F(1,18)=41.98, p<.001]$, indicating that mean judgments made from the $\delta=135 \mathrm{deg}$ viewing point were higher than those made from the $\delta=90 \mathrm{deg}$ one.

The effect of slant was significant $[F(12,96)=53.19$, $\mathrm{p}<.001$, indicating that judgments were affected by surface slant. An interaction between slant and condition was also found $[F(12,96)=6.10, p<.001]$, indicating that the relationship between judged and surface slant depended on viewing point. Accuracy differences are apparently caused by difficulties in

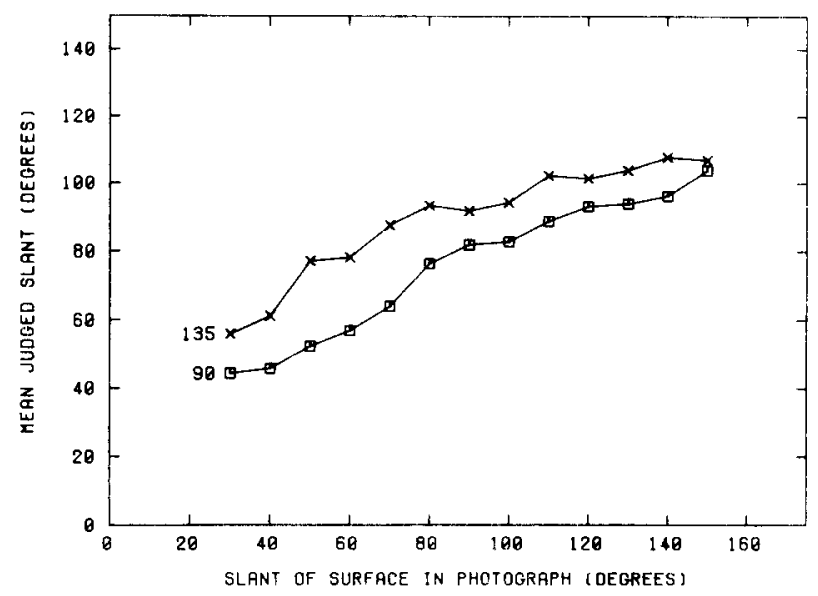

Figure 2. Judged surface orientation as a function of actual surface orientation for the two viewing conditions in Experiment 1. setting the palm board with its right side closer while using the right hand.

There was also a marginal interaction between condition and block $[\mathrm{F}(1,18)=6.10, .05>\mathrm{p}>.01]$, which arose simply because of a small practice effect (3.5 deg change in performance) at the $\delta=135 \mathrm{deg}$ viewing point.

The differences in judgment as a result in change in viewing point indicate that judged orientation was substantially affected by projected slant. Thus, it would appear that the observers were unable to discount the effects of picture plane rotation on the projected orientation. If we plot judged slant against optically projected slant (against $\theta^{\prime}$ in Equation 1), as in Figure 3, we see that performance in the two viewing conditions is virtually identical. Figure 3 shows that performance in the two conditions results in superimposed graphs, indicating that in both viewing conditions judgments were directly related to projected slant. It is not possible to compare performance as a function of projected slant statistically because of the shift in actual slant values as a result of the transform. However, simple visual comparison of Figures 2 and 3 reveals that judgments in the two viewing conditions are more closely related to projected slant than to slant of the surface depicted in the photograph.

This dependence of perceived orientation on projected texture gradient information provides partial support for the information-based theory of pictorial representation described above. If perception of space represented in a picture is determined by the information projected to a viewing point, perception should change as the information is distorted, and it does. Such a result, however, is consistent with all theories of picture perception. From any theoretical vantage, a relationship must exist between the geometric projection and perception. Clearly, the nature of the optic projection provides a necessary condition for

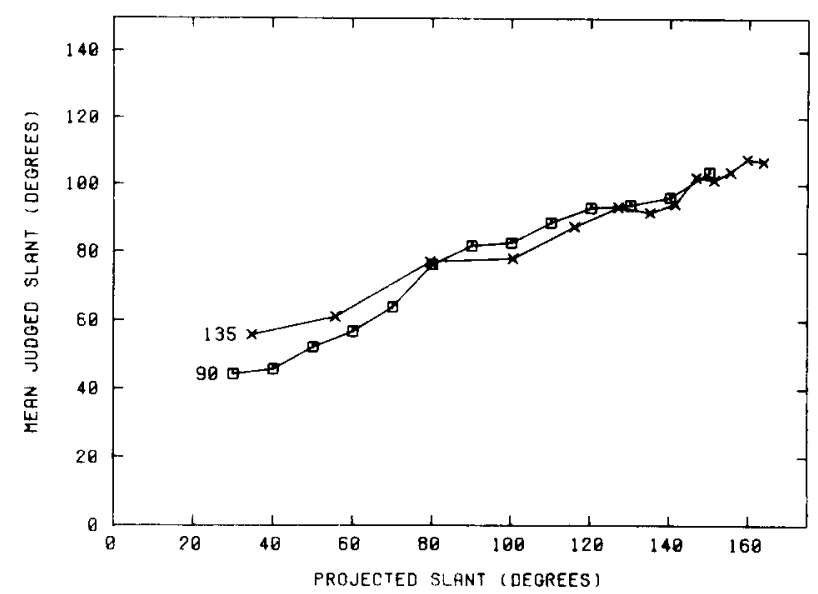

Figure 3. Judged surface slant as a function of projected surface slant in Experiment 1. 
perception. Under the present viewing conditions, all binocular, motion-carried, or assumptive "cues" were eliminated. It is not terribly remarkable, then, that perception corresponds to the projected array when only the projected array is available.

The information-based theory of picture perception rests on the claim of the sufficiency of projected information, not merely its necessity. Furthermore, the phenomenal reports of pictorial compensation provided by some (e.g. Pirenne, 1970) do not involve such strict stimulus control binocular viewing in a normally lit room is the usual situation. Under such conditions, there is ample information for the location of the picture plane. For any center of projection, the position of the picture plane is perfectly correlated with the distortion of depicted space. Under such conditions, does the projected array provide a sufficient basis for perception? Does a compensation process overcome geometric distortion caused by displacement of viewing point? Experiment 2 was conducted to address these questions.

\section{EXPERIMENT 2}

\section{Method}

Observers. Ten students (five male, five female) from the University of Pittsburgh took part in the experiment in order to satisfy a course requirement. All individuals had visual acuity of 20/40 (corrected) or better, and any who normally wore corrective lenses did so in the experiment. None of the students who served as observers in this experiment participated in Experiment 1.

Apparatus. The black matted photographs used in Experiment 1 were again used in this experiment. No viewing box was used, however. In this study, the photos were held in a black rectangular wooden frame attached to an optical bench. The height of the frame was adjusted so that the center of the photograph was at the same height as the observer's eye. Ambient fluorescent room light illuminated the stimuli.

The photographs were viewed binocularly, while the subject's head was held in position by an ophthalmic chin- and headrest. In both experimental conditions, the photos were viewed from a distance $50 \mathrm{~cm}$ away from the center of the photograph. In order to strictly assess the existence of a perceptual compensation, both viewing points were incorrect. In one condition, a line from the viewing point to the center of the photograph made an angle $\delta=135 \mathrm{deg}$ with the photographic surface; in the second condition, the line of sight intersected the picture plane with $\delta=45 \mathrm{deg}$. Other details of procedure were identical to those in Experiment 1.

\section{Results and Discussion}

The optical projections for the original surface slants for the two viewing conditions used in this experiment are depicted in Figure 4. Note that with regard to the actual surface slant depicted in the photo, both viewing conditions result in a projective distortion. This distortion is greatest $(90 \mathrm{deg})$ for a surface slant of $90 \mathrm{deg}$ (i.e., when the slanted surface is parallel to the picture plane). If array geometry was the sole determiner of perceived orientation, we should expect judged slant to reflect this distortion. The actual data are depicted in Figure 5. Although

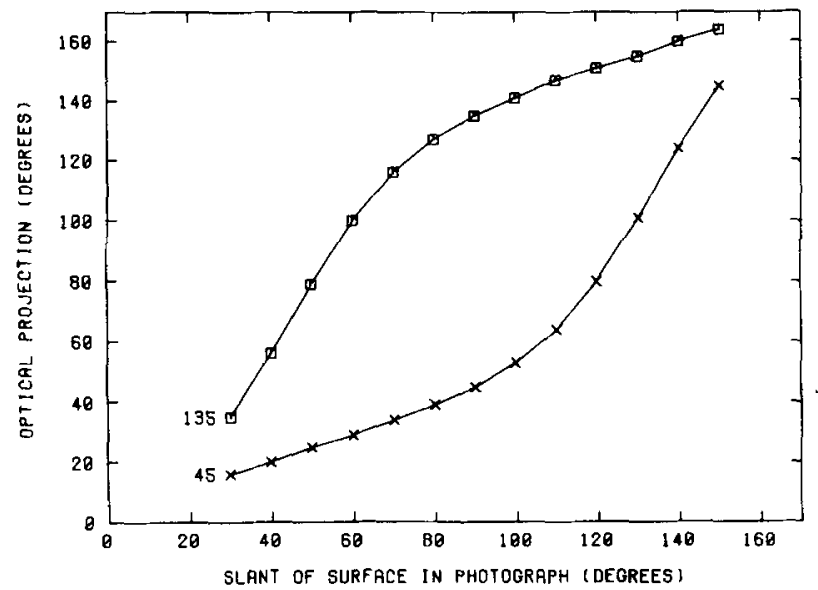

Figure 4. Projected surface orientation for the two viewing conditions ( $\delta=45 \mathrm{deg}$ and $\delta=135 \mathrm{deg})$ in Experiment 2 .

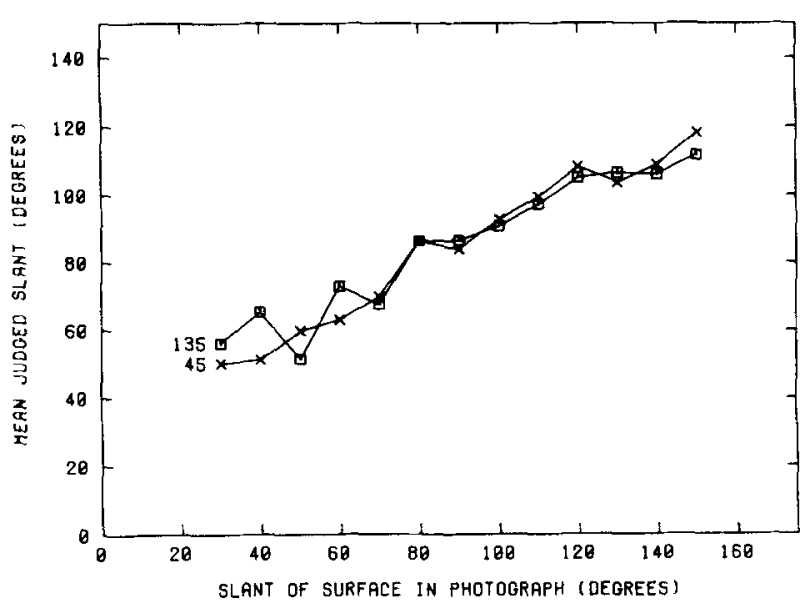

Figure 5. Judged slant as a function of physical slant in Experiment 2.

there is some variability, the two curves are virtually superimposed. These data were analyzed in a 2 (sex) by 2 (condition) by 2 (block) by 13 (slant) ANOVA with repeated measures on the last three factors. There was a significant effect of surface slant on judgment $[F(12,96)=43.59, p<.001]$, merely indicating that the orientation of the surface in the photograph affected judged orientation. However, there was no effect of viewing condition on judgment $[\mathrm{F}(1,8)<1.0, \mathrm{p}\rangle .10]$. The sizable distortion introduced in the two conditions had no affect on performance. The statistical analyses indicate that a visual compensation process is capable of overcoming the effects of projective distortion if the picture plane location is specified through full-cue binocular viewing. This implies that judged orientation of the surface in the picture is not determined simply by the optical projection. Figure 6 depicts the judged orientation as a function of projected orientation. Judgments in the two conditions plotted this way differ substantially. Projected slants are not associated with 


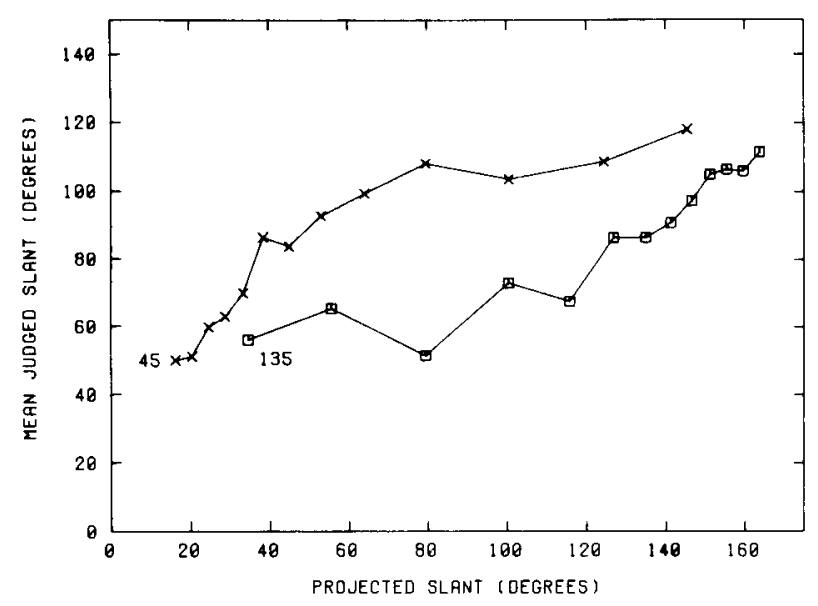

Figure 6. Judged orientation as a function of the projected orientation for the two viewing conditions in Experiment 2.

the judgments across condition. In addition to the projective information for slant, some other factor is affecting judgment. The distorted projections are not a sufficient basis for judgments of slant.

\section{GENERAL DISCUSSION}

The present experiments provide one of the first clear and unequivocal demonstrations of the existence of the phenomenon of pictorial compensation. As such, these data and the relationship between experiments have several noteworthy aspects.

First, the two experiments taken together cast doubt on the sufficiency of a direct, informationbased theory of picture perception. Earlier suggestions that the pictorial array may not be a sufficient basis for pictorial space perception led Gibson to alter his views regarding pictures. In his most recent book, Gibson (1979) suggests that there is a perceiving of the surface of the picture, coupled with an "indirect awareness" of the surface depicted in the picture. The present results can be seen as more precisely specifying the effects of pictorial viewing. In Experiment 1, judgments were in rough correspondence with optical information (reflecting, perhaps, the imprecision of texture gradient registration). When full-cue binocular viewing specified the location of the picture plane in Experiment 2 (i.e., when the stimulus object is more clearly a picture), projective distortions of stimulus information do not affect judgment.

Second, pictorial compensation as demonstrated in Experiment 2 cannot be based simply on optical information. Geometrically, there is no information that specifies the existence of any distortion or the location of the correct viewing point. A photo can be made with a view camera, for example, with a correct viewing point at an infinite number of locations. There is nothing that is geometrically unique to the ar- ray projected from such a photo that would reveal the nature and extent of any distortion.

We propose that pictorial constancy is based upon a set of assumptions regarding the nature of pictorial viewing and the location of the correct viewing point. Although the correct viewing point could be anywhere, the perceptual system presumes that it is located along a line perpendicular to the center of the picture plane. Assumptions about the location of the correct viewing point could logically form the basis of constancy. The differences between the two studies indicate that the pictorial constancy occurs only when information for the location of the picture plane is available (Experiment 2).

A model of pictorial constancy must have the following characteristics. First, constancy must be nonlinear (as indeed it is) if it is to restore linearity of judgment over a nonlinear transformation. Second, a compensation process must modulate projected information for orientation by the discrepancy between actual (registered) or assumed correct viewing point. We suggest that constancy can be modeled by some version of the distortion equation described above or by the distortions described by Farber and Rosinski (1978) or Rosinski and Farber (1980).

As shown, depicted surface slant, $\theta$, is a function of projected slant, $\theta^{\prime}$, and picture plane inclination, $\delta$. (Since the correct viewing point is assumed to be along a line perpendicular to the picture plane, $\delta$ is directly related to the discrepancy between actual and correct viewing points.) Constancy can then be modeled in terms of the visual system estimating $\theta^{\prime}$ and $\delta$ and computing $\theta$. Note that although the system must register projected slant and picture plane orientation, there is no implication that conscious awareness of these two parameters is necessary.

It is clear that the accuracy of compensation is affected by the accuracy with which these two variables are estimated. Numerous studies (see Flock, 1965) have shown that projected slant is not perfectly judged. In Experiment 1 , viewing from $\delta=90 \mathrm{deg}$ results in the projected and surface slants' being identical. Judgments are not, however, in psychophysical correspondence with surface slant. Such results may simply reflect inaccuracy in the ability to register monocular texture gradient information.

In addition, these two experiments indicate the effects of $d$ estimation. In Experiment 1, information for picture plane orientation was minimized and limited to accommodation and accommodative convergence. Such reduced viewing results in no pictorial compensation. Providing binocular, perspective, and occlusion information for the picture plane in Experiment 2 sufficiently defines $\delta$ for virtually complete compensation to occur.

In summary, although it is true that the fidelity of representation depends on information projected to 
the eye, geometric information does not provide a sufficient basis for picture perception. The visual system is able to compensate for effects of perspective distortion caused by incorrect viewing. It appears that in pictorial compensation, both projected information and the discrepancy between actual and assumed correct viewing point combine to determine perceived orientation.

\section{REFERENCES}

Farber, J., \& Rosinski, R. R. Geometric transformations of pictured space. Perception, 1978, 7, 269-282.

FLock, H. R. Optical texture and linear perspective as stimuli for slant perception. Psychological Review, 1965, 72, 505-514.

Gibson, J. J. A theory of pictorial perception. Audio-Visual Communication Review, 1954, 1, 3-23.

Gibson, J. J. The information available in pictures. Leonardo, $1971,4,27-35$.

GiBson, J. J. The ecological approach to visual perception. Boston: Houghton Mifflin, 1979.
Haber, R. N. Visual perception. In M. R. Rosenzweig \& L. W. Porter (Eds.), Annual Review of Psychology, 1978, 29, 31-59. Hagen, M. A. Picture perception: Toward a theoretical model. Psychological Bulletin, 1974, 81, 471-497.

Hochвe RG, J. The psychophysics of pictorial perception. AudioVisual Communication Review, 1962, 10, 22-54.

Kennedy, J. M. Icons and information. In D. R. Olson (Ed.), Seventy-third yearbook of the National Society for the Study of Education, Media and Symbols: The forms of expression, communication, and education. Chicago: University of Chicago Press, 1974.

Perkins, D. N. Compensating for distortion in viewing pictures obliquely. Perception \& Psychophysics, 1973, 14, 13-18.

Pirenne, M. H. Optics, painting \& photography. Cambridge, England: Cambridge University Press, 1970.

Rosinski, R. R. Picture perception and monocular vision: A reply to Hagen. Psychological Bulletin, 1976, 83, 1172-1175.

Rosinski, R. R., \& Farber, J. Compensation for viewing point in the perception of pictured space. In M. Hagen (Ed.), The psychology of pictorial representation. New York: Academic Press, 1980.

(Received for publication March 28, 1980; revision accepted June 30,1980 .) 